\title{
OFDM signal with BPSK modulated subcarriers for multi-carrier ionospheric sounding
}

\author{
Vladimir Ivanov, Natalya Ryabova, Alexey Elsukov", and Alexey Laschevsky \\ Volga State University of Technology, 424000 Yoshkar-Ola, Russian Federation
}

\begin{abstract}
Paper presents the developed algorithm for multi-carrier simultaneous vertical ionospheric sounding by OFDM signal with BPSK modulation of subcarriers. There is presented a mathematical model, numerical and simulation modeling with the use of USRP platform. Research findings proved the fundamental feasibility of using orthogonal subcarriers for simultaneous sounding of channels over a wide band.
\end{abstract}

\section{Introduction}

State-of-the-art wireless communication technologies, including cognitive radio [1], employ methods of finding optimal radio channels from the available at that time channels by means of sounding. It is especially topical for the signal propagation medium (ionosphere) that varies in time and space.

Vertical and oblique sounding used in communication and positioning systems [2]. Vertical ionosondes are typically employed to support Near Vertical Incidence Skywave (NVIS) systems [3, 4]. Currently, ionosondes utilize signals with binary phase-shift keying (BPSK) and linearly frequency modulation. Signal bandwidth defines delay resolution of the ionosonde. However, it is limited to the values of $30-40 \mathrm{kHz}$ due to the influence of frequency dispersion [5]. Hence, the energy efficiency, and consequently, the quality of the received data are ensured through the use of longer signals considering echoes integration during processing. BPSK signal duration can be increased by the coherent integration of several echoes over the channel coherence time. Multi-carrier (panoramic) sounding yields data on the frequency-ordered set of channels as well as on the structure of layered ionosphere [3]. Typical sounding procedure is performed sequentially i.e. the sounding frequency sweeps in the entire HF band. Hence, an increase in the integration time at a single carrier causes an increase in the sounding session time. An alternative approach can be a simultaneous sounding at each carrier. It can be implemented by means of Orthogonal Frequency-Division Multiplexing (OFDM) signal with BPSK for spreading the spectrum of each carrier. In this case, the sounding session time does not depend on the number of carriers and is determined only by the time of integration of the signal energy.

OFDM signals are widely used in radar [6,7]. However, sounding the ionosphere and HF communication channels requires considering the features of the problem and signal propagation conditions. The paper is addressed to the study of this problem. The developed OFDM ionosonde was implemented with the use of Universal Software Radio Peripheral (USRP) platform supported by the groundbreaking software-defined radio (SDR) technology.

*Corresponding author: ElsukovAA@,volgatech.net 


\section{OFDM signal synthesis for multi-carrier sounding}

Over the sounding session time, OFDM signal consists of $\mathrm{K}$ orthogonal subcarriers $\omega_{k}$ (where $k \in[0, K-1]$ ), that convey pulses with duration $T_{S}$ and repetition period $T_{R}$. Sounding impulse is internally modulated with BPSK and consists of M chips of duration $T_{C}$. Thus, $T_{S}=T_{C} M$ (fig. 1)

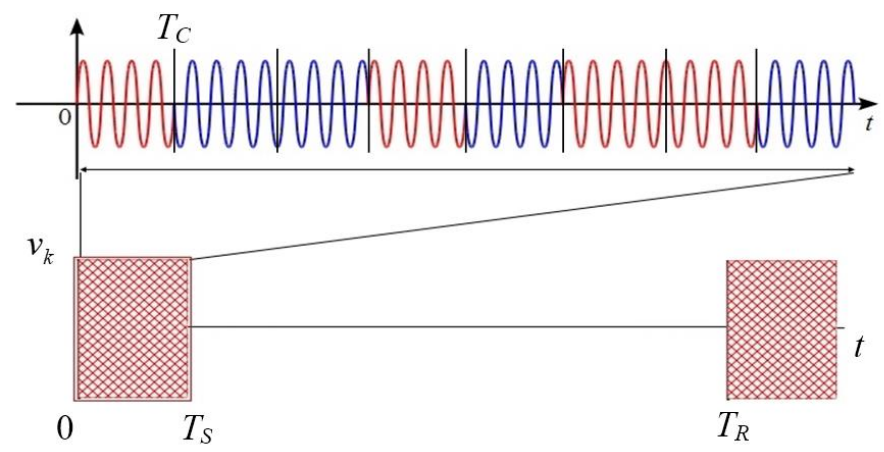

Fig. 1. Periodically repeated pulse with BPSK modulation generated at each subcarrier.

In general, complex-valued amplitude of a signal at a subcarrier can be expressed as follows:

$$
v(t)=v_{0 k}(t) \exp \left(j \varphi_{k}\right)
$$

where

$v_{0 k}(t)$ - amplitude that is greater 0 within duration $T_{S}$,

$\varphi_{k}$ - initial chip phase that is between 0 and $\pi$ values.

Such OFDM signal is represented as follows:

$$
\hat{u}(t)=\frac{1}{K} \sum_{k=0}^{K-1} u_{k}(t)=\frac{1}{K} \sum_{k=0}^{K-1} v_{0 k}(t) \exp \left(j \varphi_{k}\right) \cdot \exp \left(j \omega_{k} t\right)=\frac{1}{K} \sum_{k=0}^{K-1} \hat{v}_{k}(t) \exp \left(j \omega_{k} t\right)
$$

The minimum subcarriers spacing defines the maximum possible density of subcarriers in the frequency domain. Thus, it is determined by the orthogonality condition over a time interval equal to the chip duration:

$$
\begin{gathered}
\int_{0}^{T_{C}} u_{k}^{*}(t) \cdot u_{k+1}(t) d t=0 \\
\int_{0}^{T_{C}} u_{k}^{*}(t) \cdot u_{k+1}(t)_{k+1} d t=v_{0 k} \cdot v_{0 k} \cdot \exp \left[j\left(\varphi_{k+1}-\varphi_{k}\right)\right] \int_{0}^{T_{C}} \exp \left[j\left(\omega_{k+1}-\omega_{k}\right)\right] t \cdot d t= \\
=v_{0 k} \cdot v_{0 k} \cdot T_{C} \cdot \exp \left[j\left(\varphi_{k+1}-\varphi_{k}\right)\right] \cdot \exp \left[j\left(\omega_{k+1}-\omega_{k}\right)\right] \frac{T_{C}}{2} \cdot \sin c\left(\omega_{k+1}-\omega_{k}\right) \frac{T_{C}}{2}=0
\end{gathered}
$$

Excluding the case of equal frequencies, orthogonality occurs when the last function in (3b) is zero, i.e. when its argument equals $\pi$.

Thus, adjacent subcarriers and their spacing are as follows:

$$
f_{k+1}=f_{k}+\frac{1}{T_{C}} \text { or } f_{k+1}-f_{k}=B_{C}=\frac{1}{T_{C}} .
$$


We shall note, that it is strictly required to satisfy (4) to ensure subcarriers orthogonality. where:

If sounding frequency band equals $B, N$ subcarriers can occupy that band (fig. 3),

$$
K=\frac{B}{B_{C}}=B \cdot T_{C}
$$

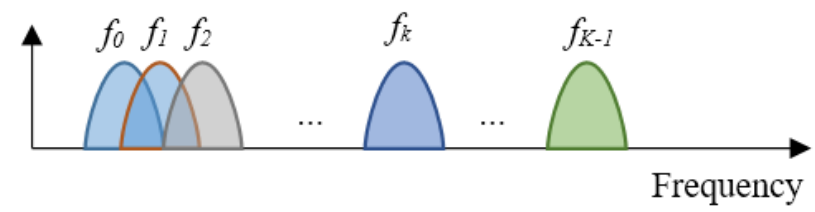

Fig. 2. Orthogonal subcarriers grid within soudning band.

Let us turn to the discrete time representation in (1) so that there are $N$ samples exist over chip duration (1). Hence, time step between samples is $d t=\frac{T_{C}}{N}$ :

$$
\begin{gathered}
t=t_{n}=n \cdot d t=T_{C} \frac{n}{N}=\frac{n}{B_{C} N} \\
\omega_{k}=k \cdot d \omega_{k}=k \cdot \frac{2 \pi B}{K}=k \cdot 2 \pi B_{c} \\
\omega_{k} t_{n}=\frac{2 \pi}{N} k \cdot n
\end{gathered}
$$

Considering discrete time, (1) can be represented as follows:

$$
\hat{u}(n)=\frac{1}{K} \sum_{k=0}^{K-1} \hat{v}_{k}(n) \exp \left[j \frac{2 \pi}{N} n \cdot k\right]
$$

It can be seen that the OFDM signal is the inverse Fourier transform of the complex amplitudes for each subcarrier. Thus, the signal generated by the given complex-valued amplitudes with the use of inverse Fourier transform (according to (7)) is a frequency grid of modulated subcarriers with spacing $f_{k+1}-f_{k}=\frac{1}{T_{C}}$.

In practical applications, using (7), the coefficient $\frac{1}{K}$ can be neglected and OFDM signal is generated according to the following equation:

$$
\hat{U}(n)=K \cdot \hat{u}(n)=\sum_{k=0}^{K-1} \hat{v}_{k}(n) \exp \left[j \frac{2 \pi}{N} n \cdot k\right]
$$

Let us derive the equations for computing samples of a total signal that is the set of samples of the function (8) in time domain. In this case, one should set up the number of a time sample and sort out samples in the frequency domain. Procedure yields a set of samples of the total signal in the time domain $\{\hat{U}(0), \hat{U}(1), \ldots, \hat{U}(n), \ldots, \hat{U}(N-1)\}$, where: 


$$
\begin{gathered}
\hat{U}(0)=\sum_{k=0}^{K-1} \hat{v}_{k}(0) \\
\hat{U}(1)=\sum_{k=0}^{K-1} \hat{v}_{k}(1) \exp \left[j \frac{2 \pi}{N} k\right] \\
\ldots \\
\hat{U}(n)=\sum_{k=0}^{K-1} \hat{v}_{k}(n) \exp \left[j \frac{2 \pi}{N} n \cdot k\right] \\
\hat{U}(N-1)=\sum_{k=0}^{K-1} \hat{v}_{k}(N-1) \exp \left[j \frac{2 \pi}{N}(N-1) \cdot k\right]
\end{gathered}
$$

It is seen that the corresponding subcarrier is absent in the grid when an amplitude of a modulating signal equals zero.

In the ionospheric radio channel, each subcarrier is multiplied by the corresponding complex-valued transfer coefficient, that can be measured by means of BPSK compression. Demodulation in the receiver yields complex-valued modulating signals at each subcarrier. For this purpose, functions $\hat{U}(n)$ are transformed to the frequency domain with the use of Fourier transform. As a result, we obtained equation to derive complex-valued amplitudes for each subcarrier in the total signal:

$$
\hat{v}_{k}(k)=\sum_{n=0}^{N-1} \hat{U}(n) \exp \left[-j \frac{2 \pi}{N} n \cdot k\right]
$$

In this case, for a certain subcarrier all samples of the total signal are sorted out by the time samples as an ordered set $\left\{\hat{v}_{0}(0), \hat{v}_{1}(1), \ldots, \hat{v}_{k}(n), \ldots, \hat{v}_{K-1}(N-1)\right\}$, where:

$$
\begin{gathered}
\hat{v}_{0}(0)=\sum_{n=0}^{N-1} \hat{U}(n) \\
\hat{v}_{1}(1)=\sum_{n=0}^{N-1} \hat{U}(n) \exp \left[-j \frac{2 \pi}{N} n\right] \\
\ldots, \\
\hat{v}_{k}(k)=\sum_{n=0}^{N-1} \hat{U}(n) \exp \left[-j \frac{2 \pi}{N} n \cdot k\right] \\
\ldots, \\
\hat{v}_{K-1}(K-1)=\sum_{n=0}^{N-1} \hat{U}(n) \exp \left[-j \frac{2 \pi}{N} n \cdot(K-1)\right]
\end{gathered}
$$


Compression of each subcarrier in the time domain yields a set of samples of the complex-valued impulse responses of the corresponding ionospheric channels at the subcarriers. Thus, we obtain several frequencies, each of which will deliver information on the parameters of the corresponding ionospheric frequency channel. Compression is performed by computing the correlation integral and applying coherent integration. It yields a power delay profile (PDP). Applying the appropriate processing allows to obtain channel parameters (signal-to-noise ratio, delay spread and Doppler spread) [4], and the frequency ordered set of PDPs produces an ionogram of vertical ionospheric sounding.

\section{Computer modelling}

In our studies, we modeled OFDM signal with 8 subcarriers that were internally modulated with BPSK. Calculations were carried out in GNU Radio Companion. Signal parameters were chosen according to the limitation of the ionospheric channel bandwidth due to the frequency dispersion of the medium (bandwidth should not exceed $30 \mathrm{kHz}$ ) [3, 8]. In our case, for technical implementation, we selected the subcarrier bandwidth of $25 \mathrm{kHz}$, so that 8 subcarriers occupied the $200 \mathrm{kHz}$ bandwidth. Each subcarrier was modulated by a Barker sequence with $\mathrm{M}=13 \mathrm{chips}$. The pulse duration was $T_{S}=520 \mu \mathrm{s}$, and the repetition period was $T_{R}=5.12 \mathrm{~ms}$.

Figure 3 illustrates the shape of the transmitted signal at a subcarrier.

OFDM signal was obtained with the use of inverse Fast Fourier Transform (FFT) and conversion of the parallel stream to a serial one. In experiments, we simulated 2 propagation modes of a signal on the path with delays of $2 \mathrm{~ms}$ and $2.3 \mathrm{~ms}$.

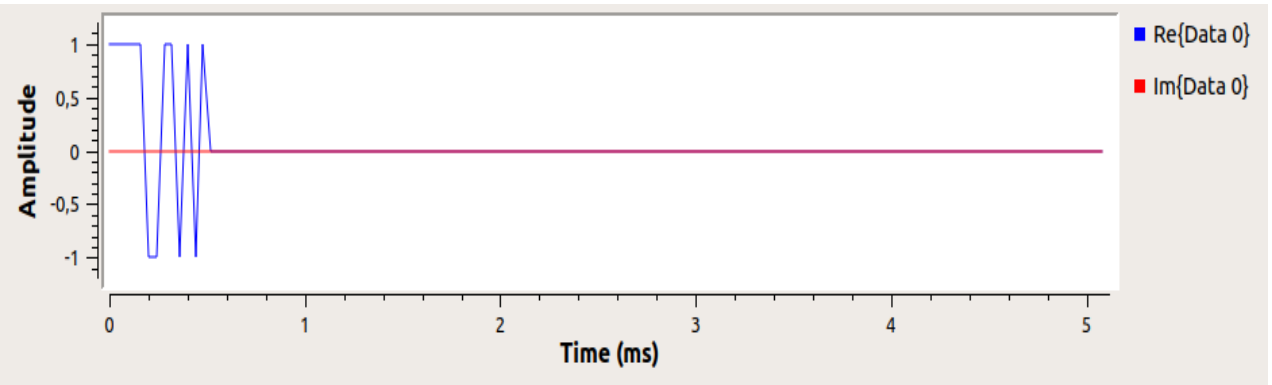

Fig. 3. Subcarrier of the transmitted OFDM signal with BPSK.

We assumed that there were the direct signal and 2 delayed signals at the receiver input.

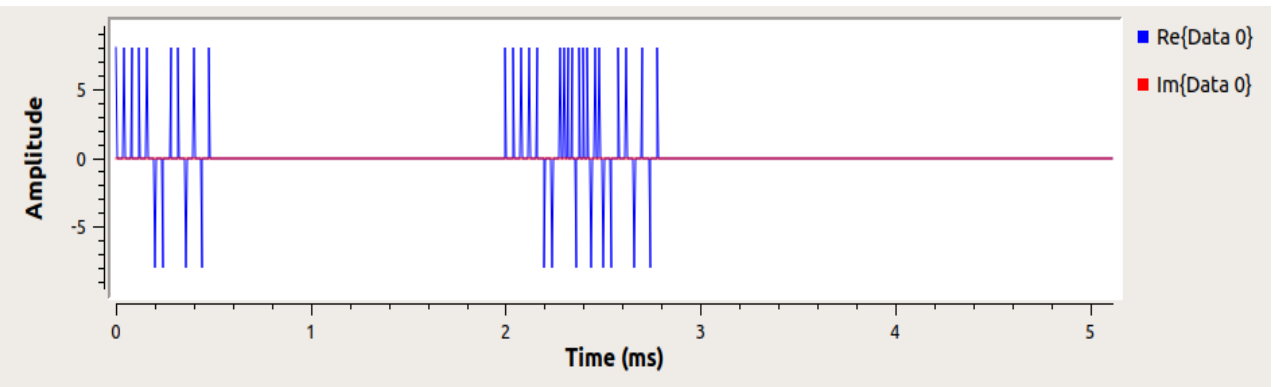

Fig. 4. Shape of the OFDM signal with BPSK at the receiver input (direct signal and 2 delayed signals).

In the receiver, subcarriers were extracted by means of direct FFT and conversion of a serial stream into the parallel one. 
Figure 5 illustrates a signal that was received at a random subcarrier.

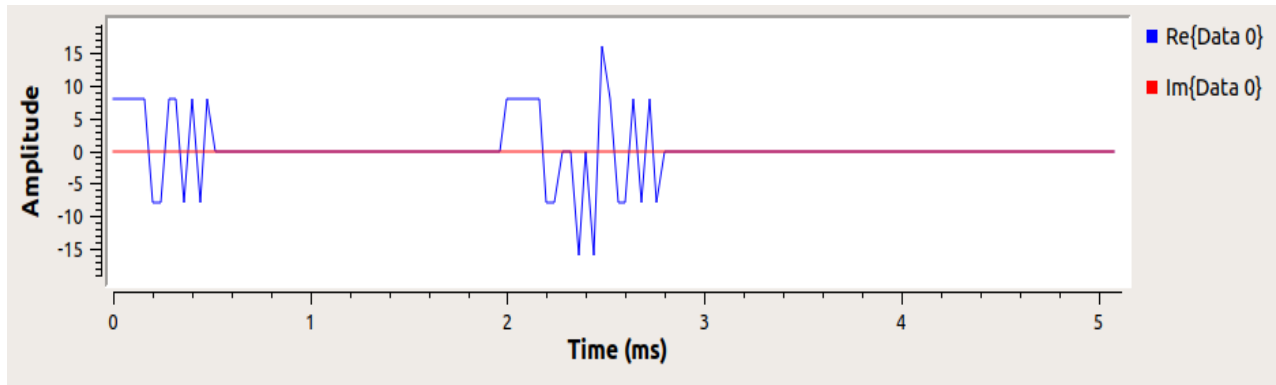

Fig. 5. Received subcarrier of the OFDM signal with BPSK (direct signal and 2 delayed signals).

PSK signal at each subcarrier was compressed by the correlation processing method.

Figure 6 presents power delay profile of the channel impulse response at the selected subcarrier.

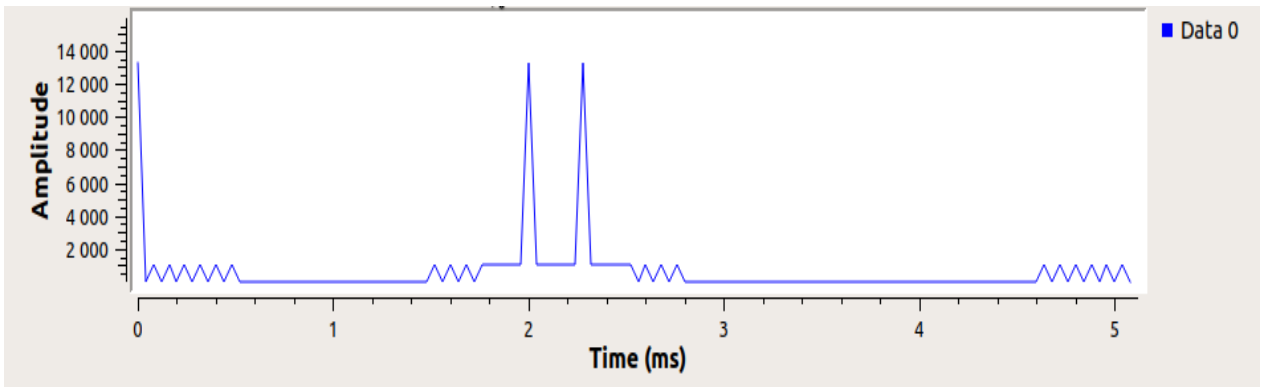

Fig. 6. PDP of the channel impulse response at the subcarrier.

\section{Simulation modelling}

Universal Software Radio Peripheral (USRP) N210 platform was used for the implementation and verification of the developed algorithm in the full-scale experiments. Fig. 7 presents the block scheme of the experimental facility.

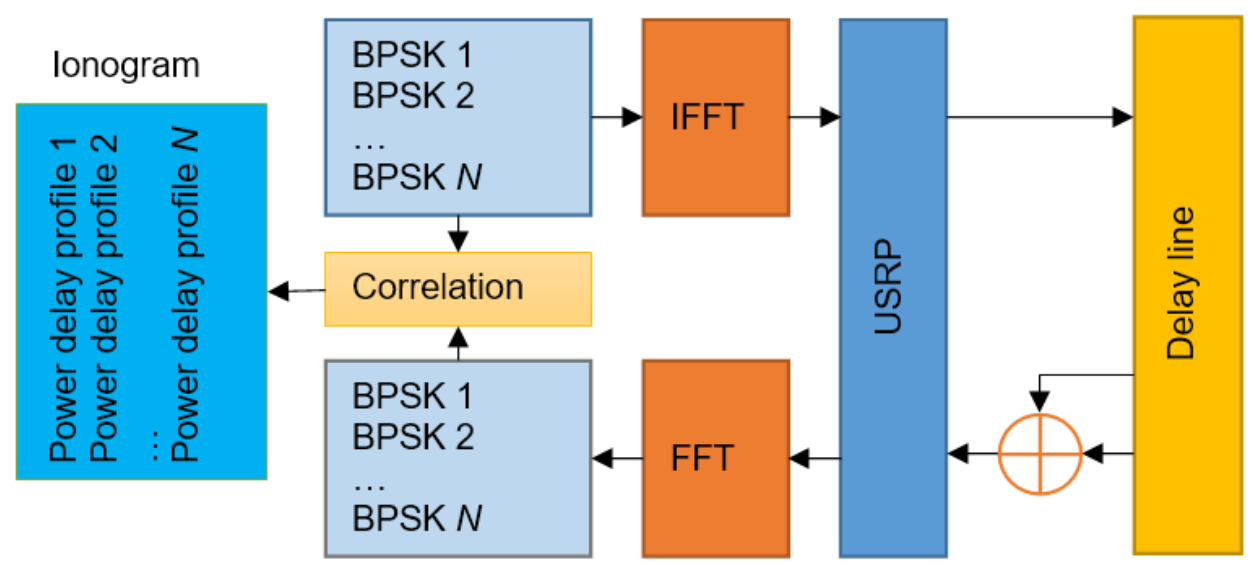

Fig. 7. Block scheme of the laboratory prototype of the OFDM ionosonde. 
In laboratory experiments (physical modeling), sounding was performed in the frequency band of 2-14.5 MHz. For this reason, according to the proposed algorithm we synthesized 512 orthogonal subcarriers with spacing $24.4 \mathrm{kHz}$. Chip duration was $41 \mu \mathrm{s}$, pulse duration was $533 \mu$ s and pulse repetition period was $5.25 \mathrm{~ms}$.

In the experiments, propagation medium was physically modeled by a dispersive tapped delay line that operates at frequency $4.6 \mathrm{MHz}$ and has bandwidth of roughly $1 \mathrm{MHz}$. It allows a signal to be delayed by two different values. First delay is $490 \mu$ s (virtual reflection height is $75 \mathrm{~km}$ ), and the other one is $730 \mu \mathrm{s}$ (virtual reflection height is $110 \mathrm{~km}$ ).

Simulation result is illustrated on the figure 8 .

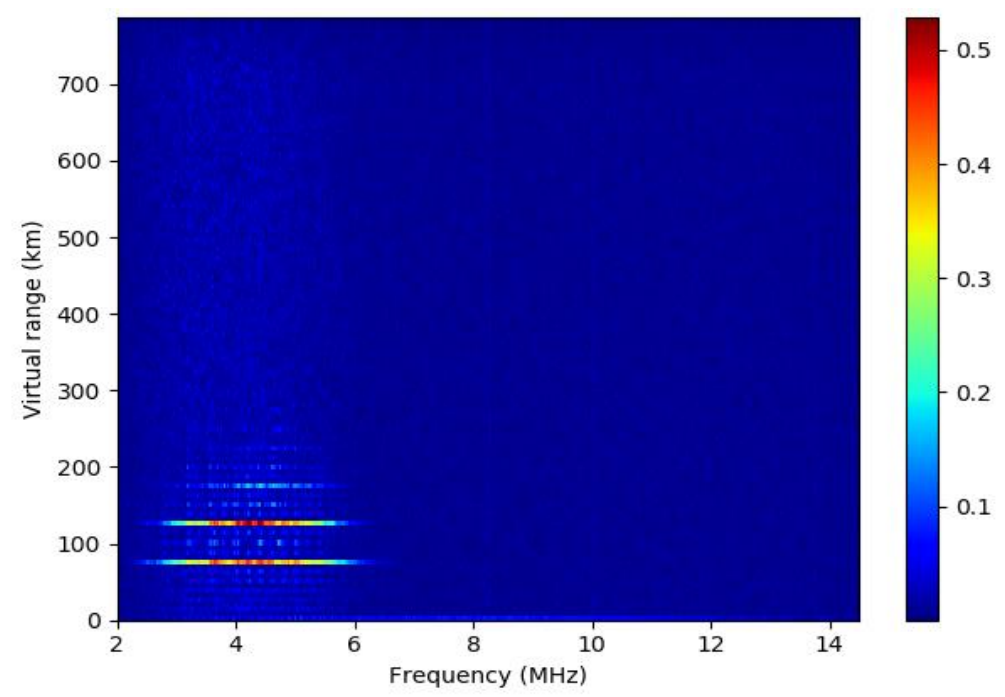

Fig. 8. Ionogram recorded in the laboratory experiments by the OFDM ionosonde.

Ionogram exhibits 2 modes that correspond to reflections of radio waves from different ionosphere layers. Frequencies of observed echoes were limited by the delay line bandwidth.

\section{Conclusions}

Research findings proved the feasibility of application of orthogonal subcarriers with BPSK for simultaneous sounding of more than a hundred channels with bandwidth of $25 \mathrm{kHz}$. Moreover, for an OFDM ionosonde, an increase in frequency resolution (frequency grid) does not affect the total sounding session time. It allows to increase the signal integration time and opens new perspectives for reducing the transmission power. Additionally, rising integration time results in an increase in the Doppler frequency resolution.

This work was supported by the grant No. 3.4074.2017/ПЧ from the Ministry of Science and Higher Education of the Russian Federation; the grants No. 19-07-00629, 17-07-00799 from the Russian Foundation for Basic Research.

\section{References}

1. T. Vanninen, T. Linden, M. Raustia, H. Saarnisaari, Cognitive HF-New perspectives to use the high frequency band, 9th International Conference on Cognitive Radio Oriented Wireless Networks and Communications (CROWNCOM 2014), Oulu, pp. 108-113 (2014) 
2. D.V. Ivanov, V.A. Ivanov, E.V. Katkov, V.V. Ovchinnikov, M.I. Ryabova, Positioning of Dynamic Objects by Means of Systems of Multi-Frequency Sounding of HF Radio Paths with the use of FMCW Signals, 2018 2nd URSI Atlantic Radio Science Meeting, (AT-RASC) paper N 8471600 (2018)

3. A.A. Elsukov, D.V. Ivanov, V.V. Ovchinnikov, N.A. Konkin, M.I. Ryabova, BPSK Ionosonde Based On SDR Platform To Support NVIS Communication, 2018 Systems of Signal Synchronization, Generating and Processing in Telecommunications (SYNCHROINFO 2018), Minsk, pp. 1-6 (2018)

4. D.V. Ivanov, V.A. Ivanov, N.V. Ryabova, A.A. Elsukov, R.R. Belgibaev, V.V. Ovchinnikov, Universal ionosonde for diagnostics of ionospheric HF radio channels and its application in estimation of channel availability, IET Conference Publications, CP741 (2018)

5. V.A. Ivanov, D.V. Ivanov, N.V. Ryabova, M.I. Ryabova, A.A. Chernov, V.V. Ovchinnikov, Studying the parameters of frequency dispersion for radio links of different length using SDR based sounding system, 2017 XXXIInd General Assembly and Scientific Symposium of the International Union of Radio Science (URSI GASS), Montreal, QC, pp. 1-3, (2017)

6. J. Fink, F.K. Jondral, Comparison of OFDM radar and chirp sequence radar, 2015 16th International Radar Symposium (IRS), Dresden, pp. 315-320, doi: 10.1109/IRS. 2015.7226369 (2015)

7. C. Sturm, T. Zwick, W. Wiesbeck, An OFDM System Concept for Joint Radar and Communications Operations, VTC Spring 2009 - IEEE 69th Vehicular Technology Conference, Barcelona, pp. 1-5 doi: 10.1109/VETECS.2009.5073387 (2009)

8. V.A. Ivanov, D.V. Ivanov, A.A Elsukov, V.V. Ovchinnikov, N.V. Ryabova, M.I. Ryabova, BPSK signal shaping and processing for digital SDR ionosonde, 2018 Systems of Signals Generating and Processing in the Field of on Board Communications, pp. 1-5 (2018) 